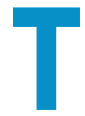

hese commentaries were received in response to a

general invitation in the February 2007 issue to encourage dissolution experts and specialists to share their opinions on the subject of mechanical and chemical calibration. The commentaries are printed in the order they were received. If other dissolution experts or specialists would like to continue the debate, please send in their commentaries and they will be published in the November 2007 issue. We appreciate the numerous responses and are pleased with the representation of ideas.

Research Editor

Dissolution Technologies 


\section{Comments on Mechanical \& Chemical Calibration by an Instrument Manufacturer}

"My dissolution tester failed the USP calibrator tablet test. What do I do?"

T his is a dreaded phone call that every instrument manufacturer has received at one time or another, perhaps more than some care to admit. Indeed, what do you do? First of all, you approach it with a rigorous, systematic procedure reviewing all critical steps in test set-up and test run. This includes a thorough review of "mechanical calibration," including paddle/basket/vessel alignment, centering, speed and temperature, vibration, and so forth. You evaluate the requirement for deaeration, as well as cleanliness and appropriateness of glassware, flow cells, and accessories. You also review what might be called the "human factor." This includes the proper execution of procedures and protocols, preparation of standards, manual sampling technique, filtration, and method of analysis. You strive to isolate variables, and by so doing, you ultimately identify the problem(s). You then establish corrective action as required.

Hanson Research manufactures 200-250 dissolution testers each year. As our equipment is designed for longevity, we have a working installed base that well exceeds 3,000 units worldwide. Some in our industry (for example, the recent ASTM Proposal E55.03) have proposed the elimination of calibrator tablets (i.e.,"reference standard tablets") in favor of a more comprehensive mechanical calibration procedure. You may think that elimination of calibrator tablets (and hence elimination of those dreaded phone calls) would make our job easier. However, I strongly support the use of calibrator tablets, which essentially constitutes "chemical calibration" of the instrument.

As an instrument designer and manufacturer, I require an industry "standard" to prove the efficacy and performance of the instrument for its intended use. Mechanical calibration is a critical component therein, as it qualifies and proves the mechanical specifications of the instrument design and its manufacture. But I also want a "performance" standard, one that qualifies the instrument in use. For dissolution testing, this requires chemical calibration with calibrator tablets.

To wit, if we designed and manufactured aircraft, I would certainly demand rigorous mechanical calibration of design specifications and operation. But I would want more. I would also require performance qualification. I would want to put that plane up in the air in a performance test to ensure it safely took off, flew, and landed as intended, before l ever allowed passengers to get on board. We call this"PQ."

This leads to a discussion of "IQ/OQ/PQ" (Installation, Operational, and Performance Qualification). IQ and OQ (often combined during execution) are essential to prove mechanical calibration and should be mandatory in every lab, for each instrument used.

Within this context, the current ASTM E55.03 proposal attempts to establish a comprehensive $\mathrm{IQ} / \mathrm{OQ}$ procedure and checklist for mechanical specifications and tolerances. This offers inherent value as it strives for a better understanding and definition of the critical aspects of mechanical calibration (although the current proposal, in my judgment, needs refinement and collaboration before it may fulfill its potential as a useful and practical tool in the dissolution lab).

However, PQ (performance qualification), in addition to $\mathrm{IQ} / \mathrm{OQ}$, must also be evaluated. $\mathrm{PQ}$ will test the instrument in use. It confirms not only mechanical calibration but actual performance in the real world (in this case, the working dissolution laboratory). PQ will monitor performance variables that mechanical calibration alone cannot. Every lab must institute training requirements for dissolution test methods and protocols. PQ can catch any lapses in training or execution; mechanical calibration cannot. PQ (chemical calibration) is therefore vital to ensure and maintain quality control.

Every instrument manufacturer, and perhaps every expert in this industry, has seen examples of calibrator tablet failures where the instrument was fine but a human mistake caused a failure. In one sense, this means calibrator tablets have done their job. Calibrator tablets have shown to be discriminating beyond mechanical problems and may also catch performance errors in a test method or protocol.

One reason for this debate is that USP calibrator tablets have not been perfect. Some are therefore considering inhouse calibrator tablets as an alternative. My primary concern here is that USP calibrator tablets, as an industrywide standard, have provided an extensive knowledge and data base. This is scientifically valuable when conducting collaborative dissolution test studies and comparing results. A common, industry-wide standard (USP) provides "applesto-apples" comparisons. A vast array of uncontrolled inhouse calibrator tablets would provide "apples-to-oranges" comparisons and may tend to constrain and limit relevant scientific inquiries.

Of our 3,000-plus dissolution testers mentioned above, a significant inventory is based in developing nations. In many such cases, dedicated and ethical scientists are working to establish viable quality control specifications for medicines, often in an environment rife with substandard and counterfeit drugs. Dissolution testing provides a relatively low cost tool for pharmaceutical quality control. In the absence of extensive and costly product testing, dissolution testing may be the only bastion of defense in providing safe medicines to local populations. Quality verification of this fundamental, basic test must not be reduced or marginalized. A full IQ/OQ/PQ regimen is paramount for ensuring ongoing drug quality control.

Royal Hanson

Hanson Research Corp. 


\section{Mechanical Calibration versus Chemical Calibration: A Debate}

\section{Introduction}

M ultiple failures have been reported worldwide for chemical calibration of dissolution baths using the two performance verification standards (earlier known as calibrator tablets), the disintegrating Prednisone RS (reference standard) tablets, and the non-disintegrating Salicylic Acid RS tablets. The underlying problem is that chemical calibration of dissolution baths is an art requiring expertise and training. Mechanical calibration, on the other hand, is a simpler and more robust alternative.

\section{Problems with Chemical Calibration}

Dissolution of Prednisone RS tablets is very sensitive to the degree of dissolved gasses in the medium. Calibration using these RS tablets requires expertise and is very time consuming. Failure is very common for first time analysts, and this adds to the frustration. Several other parameters, such as the method of tablet introduction, size and type of sampling probes, also add to the variability of the results during the calibration.

As a result of these problems, the benefits of chemical calibration are now being questioned by the pharmaceutical industry. Performance verification standards are supposed to be reliable, reproducible, and easy to use. Unfortunately, the existing calibrator tablets have failed to meet these minimum requirements for the end-users.

\section{USP Dissolution Apparatus Types}

USP has seven different dissolution apparatus that can be used for testing of various formulations. Only Apparatus 1 (basket), Apparatus 2 (paddle), Apparatus 3 (BioDisk), and USP 7 (modified USP 3 ) are required to undergo chemical calibration. None of the other apparatus have to undergo chemical calibration, even though they are designed to generate data with in-vivo relevance.

USP Apparatus 4 has been used extensively to generate biorelevant data leading to in vitro-in vivo correlation. Similarly, USP Apparatus 5 and 6 are used to evaluate transdermal systems. The data generated from these apparatus are accepted based on IQ (installation qualification), $\mathrm{OQ}$ (operational qualification), and PQ (performance qualification) performed by the manufacturer, without the need for chemical calibration.
Role of the Analyst, Instrument, and Environment

Chemical calibration using the calibrator tablets is regarded as the dissolution system suitability test. It is considered to be a test of the entire system and presumably expected to demonstrate a perfect synchrony among the analyst, instrument, and the environment. However, even if analysts are properly trained (e.g., on the technique of deaeration), analysts change over time (e.g., changes in job functions, turnover). Hence, an annual or biannual calibration procedure using the same three elements (analyst, instrument, and environment) may not be an assurance that the same three components will be the ones performing the real experiments. Moreover, calibration of the dissolution bath is no longer the responsibility of the analyst or the enduser. Instrument calibrations are now being contracted to vendors or third parties.

With regards to the instrument, it is the responsibility of the manufacturers to develop environment-independent, robust, and reliable instruments. Minimum mechanical calibration should be the only responsibility of the end-users.

Mechanical Calibration Using Vibration Measurements One justification in the past to continue with chemical calibration has been the inability to accurately measure vibration at different positions in the bath. However, commercially available tools such as centering tools, digital tachometers, depth gauges, digital thermometers, wobble meters, and accurate digital vibration sensors have left no excuse for the pharmaceutical industry to hold on the traditional, expensive, time-consuming, and non-value calibration activities.

A set of critical parameters related to the bath performance that can be routinely measured (without in-depth analyst training) and perturbed should be identified and implemented unanimously as guidelines for mechanical calibration.

Simultaneously, stringent limits should be imposed on manufacturing tolerances of dissolution baths to lessen the burden on end-users who have more important responsibilities towards patients than to doubt the integrity of their own data.

Saroj Vangani

Amgen Inc., Thousand Oaks, CA 


\section{Mechanical versus Chemical Calibration for Apparatus 1 and 2 Dissolution Equipment}

W e are willing to consider and explore alternatives to current qualification procedures for Apparatus 1 and 2 dissolution equipment that will enhance efficiency and reduce costs with the proviso that quality is not compromised. The quality added by testing calibrator tablets has been questioned. We are aware of only two instances in which results from testing calibrator tablets led to the discovery of a faulty component or configuration. In both cases, vibration was the root cause. Insufficient degassing of the medium has also been cited as a frequent cause of failures associated with testing calibrator tablets. In other cases, the tablets themselves were assumed to have been the root cause of the failure, and in these cases, more tablets were simply retested. In our opinion,"calibrator tablets" are not reproducible enough to meet our expectations of a calibration standard.

We propose a compromise or hybrid approach, whereby calibrator tablets would be used as part of operational quali- fications for new equipment or after relocation of previously qualified equipment. Periodic performance verification would follow the procedures prescribed in the current draft of the ASTM standard.

We recommend that vibration be measured directly, preferably within the dissolution vessels while the instrument is running; however, we are not aware of commercially available, certified-traceable devices for performing this measurement. We also recommend that calibration procedures specifically state what steps must be taken in the event that test results of dissolution equipment are not within the allowed tolerances.

Opinion Expressed by the Dissolution Sciences and Technology Focus Network, World Wide Pharmaceutical Development, GlaxoSmithKline

Alger Salt

GlaxoSmithKline 


\section{Mechanical versus Chemical Calibration Debate}

t would be helpful to consider the mechanical-versus-

chemical calibrator argument first, and then consider the

third "in-house" standard option. In order to evaluate the above argument, one has to look at the quality of execution of the current Calibrator Tablet tests versus the comparative value of a purely mechanical test regime.

There has been a lot of debate recently regarding the socalled Calibrator Tablets and their utility. There is no doubt that a dissolution unit operating within the current USP mechanical specifications of wobble, tool rotational speed, centering, and so on, is well able to pass both the Prednisone and Salicylic Acid Calibrator Tablet tests. This is a fact. Even so, there have been complaints that the Prednisone test, in particular, is somewhat problematic.

The point that seems to have been lost in the fog that surrounds this debate is that the current Prednisone test is not so much a confirmation of the mechanical operation of the instrument (i.e., a successful OQ), but a test of whether the operator is able to follow some simple SOP instructions and perform a dissolution experiment. It is also pertinent to mention, at this point, that if an automated system is to be tested, the component parts of the setup should also be validated. This is, again, an operator responsibility. It is the author's experience that although the dissolution tester is the obvious subject of the suitability test, the other component parts of the system, particularly the pump, are often not subject to the same level of qualification. One could write an entire book on the comparison of automatedversus-mechanical sampling and the varying influences on the results of the relevant unit processes.

The anguish that the Prednisone test seems to produce is totally unfounded. Of course, there are certain things to which this test is susceptible, in particular, dissolved air and transmitted vibration. It is therefore incumbent upon the operator to make sure, not just for this test but for all of his dissolution work, that the instrument is in an optimum setting with regard to the potential effects of external influences, which in some labs means the old fridge under the bench or the noisy pump on the BET instrument. To this extent, the introduction of a suggested vibration measurement may be helpful to some people in order to judge whether the instrument is optimally located. The level of deaeration in the medium in order to pass the test is also in the hands of the operator, and this deaeration process has to be validated by the operator in his facility. There is a mountain of information available on this subject, and you can simply use a $\mathrm{DO}_{2}$ meter to check before you start. The key word, however, is validation.

This having been said, it is clear that the adoption of a purely mechanical test regime can not provide any test of the operator and his interface with the dissolution instru- ment. It will provide a set of numbers that are of absolutely no relevance in the working environment of operator and machine.

There is, unfortunately, another variable. It has been the author's general experience that some (and not just a few) independent companies that offer both $\mathrm{OQ}$ and $\mathrm{PQ}$ tests simply do not have the experience necessary to perform this type of work. It is, therefore, up to the dissolution user to check the credibility of the outsourced supplier. So there is no real reason to do away with the Calibrator Tablet tests, but there is a very strong argument that the people who intend to carry out these tests should be well informed and be made aware of potential pitfalls; otherwise the chant of "poor tablet quality" will continue to represent an ailment for which there appears to be no cure.

This naturally has a follow-on in that there has been a debate as to whether in-house standards should be used in place of the Calibrator Tablets. From the point of view of method validation, it must be de rigueur to have such product-specific methodologies already established inhouse. Otherwise, SOPs would be random devices of no particular use. This is obviously not the case. The plethora of in-house standards that would emerge would not give anyone an overview as to whether a universal standard could be met by the large variety of Quality Control departments within the pharmaceutical industry. It is the universality of the Calibrator Tablet test that gives the test its authority and provides a clear"go/no-go" indicator, whether the operator is in Timbuktu or New England.

In conclusion, the author proposes that the current USP mechanical specifications for dissolution testers that form part of a standard OQ test are well able, if fulfilled, to afford an operator with a successful suitability test involving the current Calibrator Tablet regime. The suitability tests are not just a test of the instrument but the operator's skill and ability to carry out a relatively easy SOP. There should be more attention paid to system validation and not just dissolution tester validation. Greater attention should be paid to instrument placement and the effects of external influences such as vibration. A suggested vibration level at the vessel interface with the bath cover may be useful in identifying these potential influences. However, a bit of lateral thinking may also come in handy. In-house standards should not be adopted as a"get out of jail" option, as there would be no universally applicable standard to which we, honestly, could all conform.

John Burmicz

Marketing \& Sales Director

Pharma Test Apparatebau GmbH 


\section{USP PVT (using calibrators)}

Ded on my experience with USP calibrator tablets, I believe that the PVT test should be limited to the following situations:

1. Initial qualification $(P Q)$ of any new dissolution testers. Since we have a lot of information and data to support this, we should use Prednisone as a tool for the PQ.

2. Training of analysts on dissolution. Since prednisone has been proven to be very sensitive to physical parameters and dissolved air, this would be a perfect tool for training new analysts.

3. Investigation of OOS results. Prednisone could be used as a tool for investigating any OOS results obtained from a tester. This would aid in the elimination or confirmation of the tester as the root cause for the OOS results.

Routine calibration of dissolution testers using Calibrator tablets does not add any value since thousands of tablets in the market may not be as sensitive as prednisone. All the data generated by USP is for Prednisone, which is an atypical drug. You cannot set public standards based on an atypical drug.

I fully support the ASTM/FDA efforts to limit routine calibration to just the physical tests. I do agree that vibration is a major part of physical tests, and there needs to be a collaborative study by FDA/USP and Industry to come up with a procedure. I would suggest that $P Q R I$ should take the initiative to come up with a procedure.

I hope everyone will agree that there is no one-size-fits-all approach to all drugs that are marketed or are under development. In the new paradigm of $\mathrm{QbD}$, the responsibility for determining the critical analytical parameters should be vested on the individual company and not FDA or USP. This critical analytical parameter will be different for different drugs. Some drugs may be sensitive to dissolved air, but many others may not be. A good DOE approach to validation will help in determining the critical analytical parameter of a dissolution method.

Caffeine, anthracene, and methylparaben are some of the reference standards used by HPLC manufacturers and the industry for calibrating HPLCs. Each company has its own
SOP for HPLC calibrartion. It is important to note that you do not see any controversy about this. Just like the HPLC calibration chemical calibration for dissolution testing should be left to the discretion of the individual company.

Saji Thomas

Par Pharmaceutical, Inc.

\section{Disclaimer}

The views expressed above are my personnel opinion and in no way reflect the opinion of PAR Pharmaceutical. 


\section{Mechanical Calibration versus Calibrator Tablets}

T he debate over mechanical calibration versus the USP calibrator tablets is a healthy activity and welcomed. Distek has always supported good science and utilization of the best technique in the dissolution laboratory. Regarding the current USP calibrator test, the concept that a more stringent mechanical requirement could eventually eliminate the need for a chemical calibration would be viewed by many as a more effective way to determine apparatus suitability. This concept requires more debate and the collection of additional supporting data. Considering the years of USP calibrator tablet use and the number of dissolution units in the field, such an initiative should be undertaken with caution, and implementation should proceed with caution.

It has long been viewed that the chemical calibrator tablets were in no way representative of the customer product or a true evaluation of the apparatus. In fact, one of the most arguable points of the chemical calibrator is exactly what defines the apparatus. Fundamentally, the apparatus is viewed as the dissolution unit only. However, most would argue that one of the key components to successfully performing the test is the analyst. The importance of the role the analyst plays in a successful calibration of a dissolution unit is easily justified by the number of companies who outsource the calibration to original equipment manufactures or third-party organizations who specialize in calibrations. It is in this area where Distek believes a more stringent mechanical calibration is a step in the right direction. Implementing tighter control over specific mechanical variables should help to minimize the influence the analyst has on successfully performing a test.

Moving forward, however, we have to consider the current lab environment. There is the likely possibility that many older units would successfully pass the current USP chemical calibrator tablet test but fall outside of the proposed ASTM mechanical specifications. This has the potential to create an economic impact for many smaller and larger laboratories alike. Should the ASTM method be approved, Distek recommends that the FDA consider a "grandfather" clause by which only new units entered into service as of the effective date of the proposed ASTM method be held to the new standard. Companies with existing equipment should be allowed to choose the method that fits best within their current infrastructure and provides the best consistency within how they qualify their instrumentation. This would allow for a gradual transition toward the new methodology, minimizing the economic impact and the disruption of business to companies with older equipment.

In summary, Distek has long supported the value of meaningful chemical calibration but feels that the ASTM method proposed by the FDA is a step in the right direction. A combination of tighter mechanical specifications in conjunction with an improved or new chemical verification would be an ideal end-state. Considering the current state of the USP calibrator tablets, too much variability exists to justify this as the sole and best method for determining dissolution apparatus suitability. Distek will continue to support any initiative in the interest of good science and sound techniques and feels it is our responsibility to look at and support the continuous improvement process in the science of dissolution testing.

Jeff Seely

Sales and Marketing Manager

Distek 


\section{Requalification of USP Apparatus 1 and 2 from the Perspective of a Contract Laboratory}

$\mathrm{T}$ he basis is that all equipment successfully passed the formal $\mathrm{DQ}, \mathrm{IQ}, \mathrm{OQ}$, and $\mathrm{PQ}$ processes. From the perspective of a contract lab, the question is not whether mechanical or chemical requalification alone should be performed. Rather, a definition is needed when to perform either one or the other, or both.

Despite correct method transfers, a contract lab often has limited experience with the products under investigation. In order to correctly attribute any unexpected results to product quality, proper requalification of the analytical equipment is mandatory. The one nonroutine requalification mentioned in this commentary concerns the timepoint immediately prior to any dissolution run. For cleaning purposes, the instrument is usually partly disassembled. After the vessels or the stirring elements are remounted, requalification of the revolution speed and the temperature before starting each run is important. Additional parameters of the method may be part of a revalidation to be performed concomitantly.

Analogous to the terminology of HPLC analysis, this procedure may be called"suitability test of the dissolution assembly" and should be documented according to GMP. The dissolution assembly for which this applies consists of the bath, the sampling unit, and the transfer-dilution unit and does not include HPLC instruments or UV/VIS spectrophotometers, which have their own system suitability tests.

Concerning the periodic requalification of the equipment, a risk-based approach may also be applied for contract laboratories. At three-month intervals, a mechanical qualification is performed. It includes centering, levelness, distances, wobbling, revolution speed, and temperature. At six-month intervals, the complete mechanical requalification with the exception of the dimensions of paddle blades and vessels (but not baskets) may be performed. To detect accidental sources of vibration or other potential impacts on the outcome of the dissolution tests, the so-called chemical requalification with a reference product is an integral part of the sixmonth requalification.

For the mechanical qualification, the FDA proposal is a suitable way to go. A contract laboratory usually does not have an in-house product that might be suitable as a reference product for chemical requalification. A well-defined reference formulation such as the USP reference tablets is therefore needed. Optimum is a product that is well characterized by collaborative trials leading to a consensus value usually described statistically by confidence intervals. The laboratories participating in these pivotal collaborative trials should have GMP status.

USP Apparatus 3 and 4 require different requalification procedures.

Dr. Johannes Kraemer

Geschaeftsfuehrer / CEO

PHAST GmbH 


\section{Dissolution Systems: Mechanical or Chemical}

F or a long time, industry has used USP prednisone and salicylic acid tablets to calibrate dissolution systems. Each lab has followed a loose outline of a method to perform the tests to USP specifications; this has given way to greater variability in the way the dissolution baths are calibrated from one laboratory to the next. For instance, one laboratory may use a filter that was randomly chosen, whereas another laboratory may have chosen the optimal filter based on a filter evaluation. During an investigation of why these laboratories obtained different results, it was found that the filter choice itself resulted in over $5 \%$ difference in percent recovered. Other method variables, such as how to properly fill the dissolution vessels (weight vs volume vs heated volume), where to pull samples, and how to introduce the tablets into the vessels, could have similar effects. These are just a few examples of details not provided by the method outline that need to be addressed by each testing laboratory.

Due to the ambiguity of the method and seemingly random failures of the calibrator tablets, ASTM is proposing a new approach to dissolution system calibration, which is to use mechanical checks in place of the prednisone and salicylic acid tablets. The approach is a great idea in that the methodology would be less variable from lab to lab. However, the internal vibration of the system is one significant aspect that is not controlled by the ASTM approach and may be one of the most important checks made on the dissolution system.

External vibration of the dissolution system is caused by sources such as a mechanical shaker on the same bench top or a vortex instrument sitting next to the dissolution bath. External vibration can be measured effectively, and thus parameters can be established to control the vibration from interfering with the testing. However, the instrument used to measure the external vibration takes its measurements from the face plate only. The instrument is unable to measure vibration that is carried much like a pulse through the shafts into the media. To put it another way, during testing there is the constant force of paddle rotation within the vessel. This creates the 'current' that will erode the tablet/capsule. This can be clearly seen during the prednisone calibrator tablet test where the tablet becomes a cone centered under the rotating paddle. The internal vibration of the system creates a new force, a pulse, that pushes from the shaft out to the sides of the vessel. The end result of this pulse can also be clearly seen during the prednisone test by the formation of a ring around the cone in the middle of the vessel. This is an indication of a high failure of the prednisone test. If one were to gently touch the shaft during rotation, the vibration could be felt. But touch is not a solid control of internal vibration. No one can justify a failure because there is a lot of vibration. We are a scientific community in a heavily regulated field that needs defined instrument parameters to clearly show that the instrument is performing correctly and the testing performed with the instrument is accurate. The best test for the measurement of this internal vibration is the use of the prednisone tablets.

What if we ignore the internal vibration, skip the prednisone tablet test, and go with just the mechanical checks? In brief, here is one quick scenario of what could happen. Lab A relies on the mechanical checks only. All baths are calibrated and put into service. A dissolution method for product $X Y Z$ is being validated. Two different analysts run the sample precision portion on different days using different baths. The first analyst obtains results that are all near $100 \%$ released at the specification time point. The second analyst obtains results that are all near $93 \%$ released at the specification time point. The two analysts are outside of the protocol, which states the difference in the mean values obtained from each analyst must be NMT 5.0\%. An investigation is initiated and several days are spent trying to determine what went wrong. When compared with past data, the results seem to be correct at $93 \%$. How did the first analyst obtain higher results? Was internal vibration the cause of the uncharacteristic higher results? If so, what other data were impacted by the internal vibration of the system? Did some products pass when they should have failed? These are all questions that quality assurance will look into as they conduct an extensive investigation into all tests run by the errant dissolution bath.

The industry has come to a crossroad. There are two different methods proposed by two different standardsetting bodies. Which road should we follow? In the end, every company will need to determine the best road for itself. One way forward could be to take the middle road and combine the two methods. The mechanical checks would ensure tighter uniformity in system setup among labs. This would make it easier to transfer methods between laboratories, and the calibrator tablets would continue to offer a check for the internal vibration of the system itself. When we can accurately measure the internal vibration of the system through a mechanical instrument, we can then discuss phasing out the prednisone and salicylic acid calibrators. Until then, we need to focus on setting up a tighter method to use with the calibrator tablets to reduce the lab-to-lab variability in dissolution system calibration.

Jonathan Kretz

Watson Pharmaceuticals, Inc. 


\section{Concerns over ASTM E2503 Standard on Dissolution Apparatus Qualification}

R ecently the ASTM E55.03 Technical Subcommittee on Pharmaceutical Standards has approved the new standard E2503 entitled; Standard Practice for Qualification of Basket and Paddle Dissolution Apparatus. This document suggests the implementation of a mechanical calibration procedure for USP Apparatus 1 and 2 and the subsequent elimination of the requirement for chemical calibration with USP dissolution calibration tablets. While the advantages of a mechanical qualification procedure are apparent, the following comments have been provided to support why the ASTM Standard E2503 is not ready for practical implementation in a GMP environment at this time.

Presently several critical parameters affecting the performance of the dissolution apparatus have not been fully addressed in the new ASTM procedure:vibration, vessel and basket condition, and combined perturbation. Another concern deals with conflicting legal standards: the USP General Chapter $<711>$ on Dissolution requires verification with USP Prednisone and Salicylic Acid tablets. Conversely, the ASTM procedure states that "further calibration with dissolution calibrator tablets is not necessary."

In the views of this author, these points serve to demonstrate why the ASTM E2503 standard is not acceptable at the present time and is not capable of showing a superior ability to qualify the dissolution apparatus to the point where it better than the present mechanical procedure augmented with the USP Periodic Performance Test. Until the completion of ongoing studies to ensure the mechanical integrity of the dissolution system and its environment, we should maintain the present performance verification system with the USP Performance Verification Tablets. In the present GMP environment, we cannot afford to abandon an established qualification system while we wait for further studies and specifications to be developed in the following areas.

Vibration A vibration study is currently underway within the ASTM E55.03 Technical Subcommittee on Pharmaceutical Standards. Progress is being made with the initial studies performed on a single dissolution apparatus to understand and quantify vibration with a meaningful specification. However, the E2503 standard does not provide a mechanical method or consistent location of measurement to detect or quantify the influence of internal or external vibration on the dissolution apparatus. Vibration is one of the primary reasons for implementation of the USP tablet calibration program in 1978 , and at this time, vibration is still not well understood and is not consistently quantified. The increase in dissolution release rates due to vibration is widely known and documented and has been the source of aberrant dissolution data. Additionally, the outcome of a previous collaborative study on mechanical calibration by the PhRMA Subcommittee on Dissolution (Pharm. Forum $2000,26(4), 1149)$ stated, "it appears that some type of cali- brator tablet should be maintained until enhanced mechanical calibration is further defined (e.g., Establishing a definitive vibration tolerance)."A principal PhRMA recommendation was to "generate additional data on vibration effects." Data should be collected during mechanical calibration in an effort to establish a relevant tolerance. At this point, how will the influence of vibration be determined without a physical measurement or use of a holistic performance verification procedure?

Vessels The vessel may contribute to variability in the dissolution system. Visual inspection is subjective and does not provide assurance that the vessel will produce results consistent with a true hemispheric vessel free of defects. The standard contains little consideration for an actual hemispheric tolerance or even cleanliness of vessels. Such things have been investigated and identified as the source of calibration failures in the past. Assuming that all dissolution vessel internal specifications are consistent worldwide is alarming, especially since many methods of vessel construction produce inconsistent hemispheric shapes that greatly contribute to altered turbulence within the dissolution vessel.

Cumulative Perturbation Most of the perturbation studies to date have been performed by tuning a dissolution apparatus as close to perfect as possible and varying one parameter at a time to study its effect. If many of the mechanical parameters are actually near their tolerance limits, what will be the effect on the dissolution rate? The ideal dissolution environment is a quiet, symmetrical, smoothly rotating system. If vessel asymmetries, vibration, wobble, temperature, and spindle height are within tolerance but near the limit, would they not have a cumulative effect on dissolution rates? Dissolution failure is not always due to a single parameter.

Baskets While the standard mentions the condition of the basket, ongoing dimensional checks are warranted. A corroded basket will still be a 40-mesh basket, but the wire diameter will become out of tolerance over time. The Japanese basket is a 36-mesh basket, and it is nearly impossible to tell the difference by looking at it. The proposed mechanical method only assumes that USP/EP basket configurations are used, but there is no way to check them. Additionally, the USP basket shaft with clips is not interchangeable with an unofficial o-ring design. The o-ring attachments have exhibited a $12 \%$ lower value than the USP design, and this has not been considered in the ASTM standard.

Conflicting Legal Standards Most standard operating procedures (SOPs) and dissolution test methods used by pharmaceutical manufacturers reference established dissolution and drug release test standards contained under USP General Test chapters $<711>$ and $<724>$, respectively. Such 
SOPs state that a dissolution test is performed in accordance with USP General Test chapter with the exception of shaft speed, medium composition, Q-value, length of test, and so on. Such a statement infers adherence to the content of the General Test chapter in its entirety including a statement requiring calibration with the USP Calibrator tablets under the Apparatus Suitability Test section. The ASTM E2503 standard, which allows a mechanical calibration surrogate in lieu of the established qualification with the USP Performance Verification Tablets, will cause conflict within the present pharmaceutical QC environment regarding which qualification method to follow. Furthermore, some laboratories may actually perform both to capture aspects of the mechanical procedure that are questionable; this will unfortunately add to the existing laboratory workload.

In summary, are we comfortable qualifying the dissolution apparatus without the use of the established USP performance qualification test? In the spirit of Analytical Instrument Qualification (AIQ), the pharmaceutical laboratory is responsible for establishing a process "ensuring that an instrument is suitable for its intended application." Additionally, the Performance Qualification (PQ) tests"are usually based on the instrument's typical on-site applications and may consist of analyzing known components or standards. The tests should be based on good science and reflect the general intended use of the instrument."

These points and others were submitted to the E55.03 Technical Committee on Pharmaceutical Standards during the development of ASTM standard E2503 and have been found "non-persuasive." Without a holistic performance verification test with a performance verification tablet and knowing that vibration and vessel dimensional conformity alone have enhanced the turbulence in a dissolution vessel to a point where dissolution results are elevated, are we prepared to release to the field sub performance tablets that may have been influenced to "pass" the dissolution specification?

Bryan Crist

Scientific Affairs Manager

Varian, Inc.

\section{References}

1. Bansal, S. K.; Layloff, T.; Bush, E. D.; Hamilton, M.; Hankinson, E. A.; Landy, J. S.; Lowes, S.; Nasr, M. M.; St. Jean, P. A.; Shah, V. P. Qualification of Analytical Instruments for Use in the Pharmaceutical Industry: A Scientific Approach. AAPS PharmSciTech 2004; 5 (1), Article 22.

2. Collins, C. C. Vibration: What Is It and How Might It Affect Dissolution Testing? Dissolution Technol. 1998, 5 (4), 16-18.

3. Crist, B.; Spisak, D. Evaluation of Induced Variance of Physical Parameters on the Calibrated USP Dissolution Apparatus 1 and 2. Dissolution Technol. 2005, 12 (1), 28-31.

4. Gray,V.; Beggy, M.; Brockson,R.; Corrigan, N.; Mullen, J. A Comparison of Dissolution Results Using O-Ring vs. Clipped Basket Shafts. Dissolution Technol. 2001, 8 (4), 8-11.

5. Dissolution Testing of Immediate Release Solid Oral Dosage Forms; Guidance for Industry; U.S. Department of Health and Human Services, Food and Drug Administration, Center for Drug Evaluation and Research, U.S. Government Printing Office: Washington, DC, August 1997; p A2.

6. United States Pharmacopeia and National Formulary USP 30-NF 25; The United States Pharmacopeial Convention, Inc.: Rockville, MD, 2007.

7. United States Pharmacopeia. USP Prednisone Tablets RS Lot O0C056, USP Dissolution Calibrator, Disintegrating Type; Specification Sheet; United States Pharmacopeial Convention, Inc.: Rockville, MD, December 6, 2004.

8. Subcommittee on Dissolution Calibration, Pharmaceutical Research and Manufacturers Association (PhRMA). Dissolution Calibration: Recommendations for Reduced Chemical Testing and Enhanced Mechanical Calibration. Pharm. Forum 2000, 26 (4), 1149-1166.

9. United States Pharmacopeia. <1058> Analytical Instrument Qualification. Pharm. Forum 2006, 32 (6), 1784-1794. 